\title{
Analytical Studies on Neurocirculatory Asthenia and Related Diseases Using Psychological and Cardiovascular Parameters
}

\author{
Koichi Nagasawa, M.D., Yasusuke Gotoh, M.D., Nobuko Irda, M.D., \\ and Eiichi Kimura, M.D.
}

\begin{abstract}
Summary
In order to analyze the nature of the cardiovascular complaints in neurocirculatory asthenia (NCA) and related diseases, 83 cases were studied on the basis of the results of psychological tests (Cornell Medical Index and Yatabe-Guilford test) and cardiovascular and respiratory parameters. Almost all of the patients with normal psychological test results had abnormalities in one or more of the following: blood pressure, pulse rate, cardiac index, $T_{I I}$ in the standing position, and cardiothoracic ratio. The data suggest that the cardiovascular complaints of the normal psychological test group can be attributed to somatic factors, especially to a small size of the heart. On the other hand, the group with psychological abnormalities had hearts of normal size, a less marked lowering of $T_{I J}$ in the standing position, but markedly abnormal respiratory patterns. This indicates that the respiratory abnormalities seen in this group are likely to be the symptoms of cardiac psychoneurosis.
\end{abstract}

\section{Additional Indexing Words:}

Neurocirculatory asthenia Cardiac neurosis Small heart syndrome Psychological test Cornell Medical Index YatabeGuilford test Respiratory abnormalities

7 HE term neurocirculatory asthenia (NCA) has been applied to the condition in which cardiovascular complaints such as chest pain, dyspnea, palpitation or dizziness are present, but without underlying organic cardiac disorders. In general, these complaints are not stable, but fluctuate from day to day. According to Christie, " the term " respiratory neurosis " is somewhat preferable to NCA because the incidence of respiratory complaints is

From the Department of Internal Medicine, Nippon Medical School, Tokyo.

Address for reprint: Koichi Nagasawa, M.D., Department of Internal Medicine, Nippon Medical School, 1-1-5 Sendagi, Bunkyo-ku, Tokyo 113, Japan.

Received for publication June 6, 1981.

Manuscript revised September 4, 1981. 
higher than that of cardiovascular complaints. One of the present authors pointed out that respiratory abnormalities were frequently observed in NCA as one of the main objective findings, ${ }^{2)}$ and also reported that markedly unusual movement of the diaphragm was discovered during cineroentgenography. ${ }^{3)}$

Essential (orthostatic) hypotension and small heart syndrome ${ }^{4}$ have been regarded as being similar to NCA because prominent cardiovascular complaints are present, despite the absence of organic cardiac involvement. If these complaints can be explained by hypotension or a small heart, the term NCA would be inappropriate.

Although the etiology of cardiac neurosis may be different from NCA, it is difficult to differentiate it from NCA accurately. ${ }^{5)}$ It would be useful if an objective way of differentiating the two diseases could be found. In this respect, studies of the psychological state and personality of the patients, using the Cornell Medical Index (CMI) ${ }^{6}$ and the Yatabe-Guilford (Y-G) test, may be of great value for differentiating NCA from related diseases. However, few reports of studies on the psychological aspects of NCA have been published. The purpose of this paper was to analyze the relationship between psychological tests and the cardiothoracic ratio, electrocardiographic findings and respiratory state.

\section{Materials and Methods}

\section{(1) Subjects}

The subjects of the study were 83 patients suffering from cardiovascular complaints. Physical examination, chest roentgenogram, electrocardiogram, and other examinations revealed no abnormal findings. Thirty-one cases were male, ranging in age from 18 to 57 years (average 29 years), and 52 were female, ranging in age from 15 to 57 years (average 27 years). Cases displaying various kinds of arrhythmias, abnormal electrocardiograms, anemia, thyroid disease, pulmonary tuberculosis, and pumonary emphysema were excluded. Those with hyperventilation syndrome were also excluded because this condition is considered to be different from NCA. ${ }^{71}$ All the patients were examined by psychiatrists in order to rule out manic-depressive diseases and anxiety neurosis.

(2) Methods

1. Psychological tests

The results of the CMI were classified into 4 groups, I, II, III, and IV, using the discriminative chart of Fukamachi. ${ }^{8)}$ The results of the Y-G test were divided into 5 types, A, B, C, D, and E. In general, the CMI Groups 
I and II were considered as normal and Groups III and IV as neurotic. The Y-G test types A, G, and D were considered as normal and types B and $\mathrm{E}$ as abnormal, since the former three were regarded as being in an emotionally stable state, and the latter two as being unstable.

2. Cardiothoracic ratio (CTR)

The CTR (\%) was measured from chest roentgenograms taken in the deep inspiration phase in the standing position. Source-to-film distance was set at $150 \mathrm{~cm}$.

\section{Electrocardiogram}

Since lowering or inversion of the $T$ wave in lead II ( $T_{\text {II }}$ ) in the standing position is frequently observed in patients with NCA, this finding was used as one of the parameters. After recording a standard 12-lead electrocardiogram in the supine position, the patients assumed an active upright posture and further electrocardiograms were taken immediately and $1,3,5,7$, and $10 \mathrm{~min}$ after standing. The lowering of $T_{\mathrm{II}}$ was evaluated by comparing the amplitude measured in the supine with the minimum amplitude measured in the upright position.

4. Respiratory findings

Respiratory curves were recorded using Sanborn's metabulator. According to the criteria proposed by Kimura and Takahashi, ${ }^{21}$ the following parameters were measured as indices of NCA: (i) augmentation of the depth of respiration, (ii) irregularity in the depth of respiration, (iii) irregularity in the respiratory cycle, (iv) non-linearity of the respiratory level, and (v) amplitude of sighing.

5. Cardiovascular parameters

Cardiac output (CO) was calculated from the dye dilution curve recorded by the earpiece method and using Hamilton's formula. Cardiac index (CI) was calculated as $\mathrm{CO}$ divided by body surface area. Systolic blood pressure (BPs) was measured by Korotkov's method. Mean blood pressure (BPm) was calculated by the formula: diastolic pressure $+1 / 3$ pulse pressure; and systemic vascular resistance (SVR) by the formula: SVR $\left(\mathrm{dyn} \cdot \mathrm{sec} \cdot \mathrm{cm}^{-5}\right)=$ $\mathrm{BPm} \times 79.92 / \mathrm{CO}$. These cardiovascular measurements were obtained in the supine position, and both immediately and after 10 minutes of passive standing using a tilting table.

\section{Results}

(1) Psychological tests

As shown in Table I, 35 out of 83 cases $(42.2 \%$ ) were diagnosed as normal and 12 cases (14.4\%) as abnormal by both CMI and Y-G test. Thir- 
Table I. Relationship between Cornell Medical Index (CMI) and Yatabe-Guilford Test (Y-G)

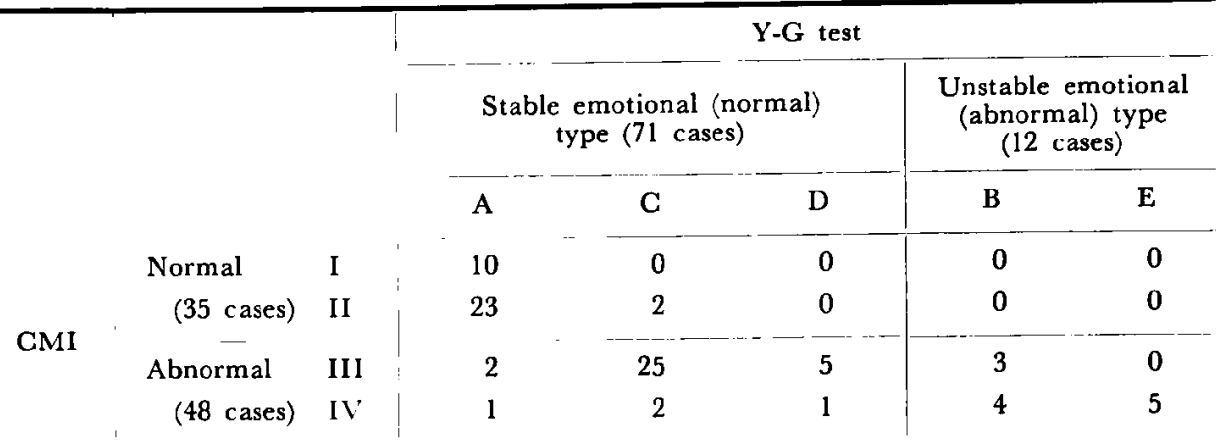

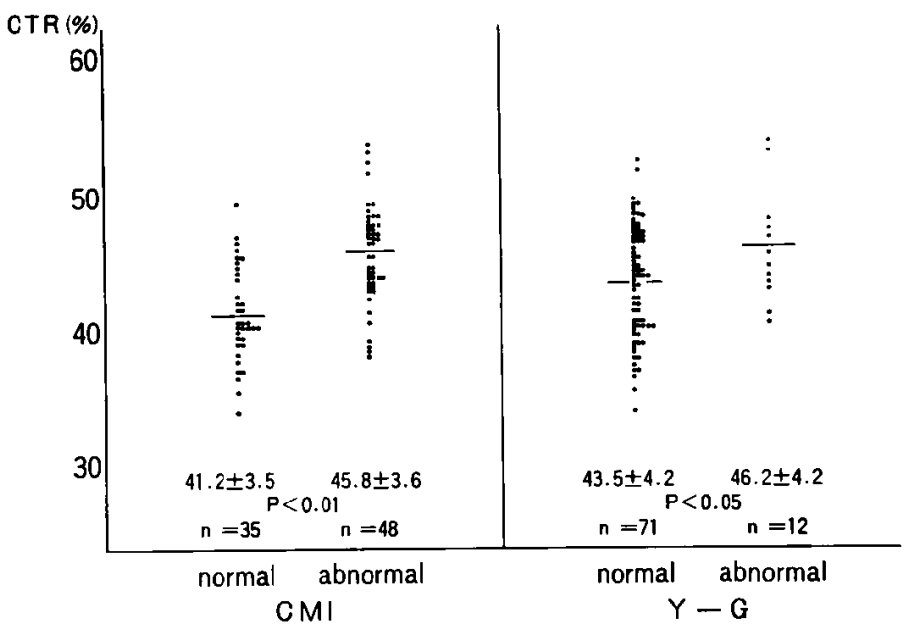

Fig. 1. Relationship between psychological tests and cardiothoracic ratio (CTR).

ty-six cases $(43.4 \%)$ who were diagnosed as abnormal by CMI showed normal Y-G test results. There was no case with a normal CMI and an abnormal Y-G test result.

(2) Relationship between heart size and the psychological tests

The average CTR of the normal CMI group was $41.2 \pm 3.5 \%$, which is much smaller than the normal heart size, while the abnormal CMI group had an average value of $45.8 \pm 3.6 \%$, which is within the normal limit (Fig. 1). There was a statistically significant difference between the CTR of the normal and abnormal CMI groups $(\mathrm{p}<0.01)$. In addition, 12 out of 16 cases $(75 \%$ ) with a CTR of less than $40 \%$ showed normal CMI results. Y-G test results also revealed a statistically significant difference $(p<0.05)$ between the CTR of the normal $(43.5 \pm 4.2 \%)$ and abnormal groups $(46 \pm 4.2 \%)$. All 


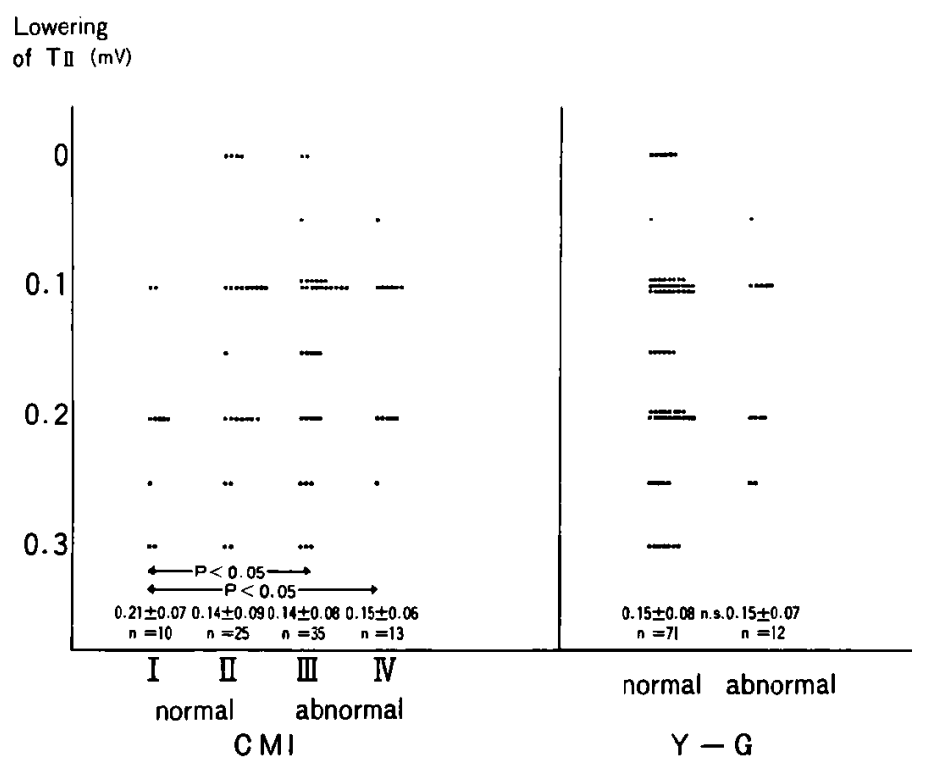

Fig. 2. Relationship between psychological tests and lowering of $\mathbf{T}$ in lead II $\left(T_{I I}\right)$ in the standing position.

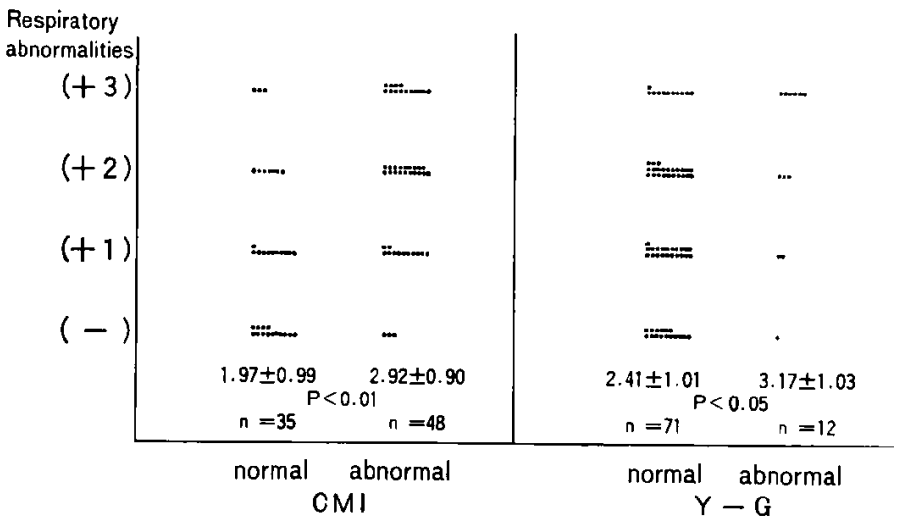

Fig. 3. Relationship between psychological tests and abnormalities in the respiratory curve. $(-)=$ normal respiratory curve; $(+1)=1$ abnormality; $(+2)=2$ abnormalities; $(+3)=3$ or more abnormalities.

of the 16 cases with a CTR of less than $40 \%$ showed normal Y-G test results.

(3) Relationship between the lowering of $\mathrm{T}_{\mathrm{II}}$ in the standing position and the psychological tests

Lowering of $\mathrm{T}_{\mathrm{II}}$ in the standing position was seen in 77 patients. As indicated in Fig. 2, the lowering of $T_{\mathrm{II}}$ was significantly more marked in the CMI-I group than in the C.MI-III and IV groups $(\mathrm{p}<0.05)$. On the other 
hand, no statistically significant difference was found between the lowering of $\mathrm{T}_{\mathrm{II}}$ in the normal and abnormal Y-G groups.

(4) Relationship between respiratory curves and the psychological tests

Among the 83 patients studied, augmentation of the depth of respiration was observed in 10 patients, irregularity in the depth of respiration in 61 patients, irregularity in the respiratory cycle in 39 patients, non-linearity of the respiratory level in 3 patients and sighing in 13 patients. Respiratory curve findings were graded for numerical comparisons as follows: 0 means no abnormalities, +11 abnormality, +22 abnormalities, and +3 more than 3 abnormalities. Fig 3 indicates the relationship between respiratory abnormalities and the results of CMI. The incidence of respiratory abnormalities was significantly higher in the abnormal CMI groups than in the normal CMI groups $(p<0.01)$. A significant difference was also noted between the incidence of respiratory abnormalities in the normal and the abnormal Y-G groups $(\mathrm{p}<0.05)$.

(5) Relationship between each pair of cardiovascular, respiratory and psychological parameters

Fig. 4 shows the relationship between the lowering of $T_{I I}$ in the standing position and CTR. A significant negative correlation was observed ( $p<0.01)$, indicating that the smaller the heart size, the more marked the lowering of $\mathrm{T}_{\mathrm{II}}$ in the standing position. Twelve out of 16 cases $(75 \%)$ with CTR of less than $40 \%$ showed lowering of $\mathrm{T}_{\mathrm{II}}$ of more than $0.2 \mathrm{mV}$ in the standing position.

A significant correlation was also observed between the GTR and the incidence of respiratory abnormalities (Fig. 5). In other words, the incidence of respiratory abnormalities was lower in patients with smaller hearts. The

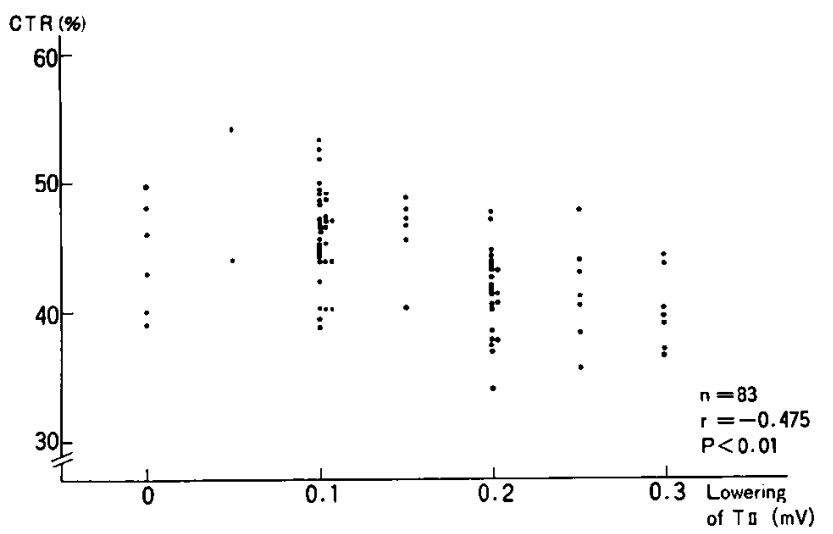

Fig. 4. Relationship between the lowering of $T_{I I}$ in the standing position and CTR. 


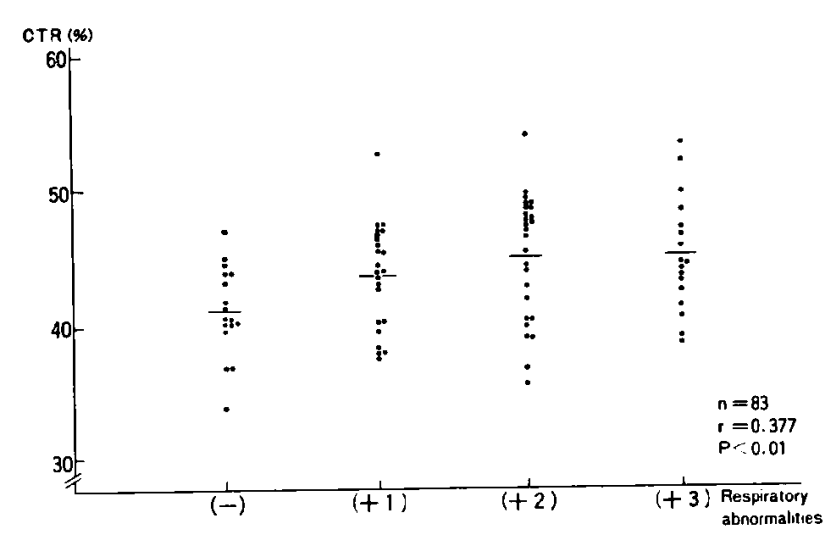

Fig. 5. Relationship between CTR and abnormalities in the respiratory curve.

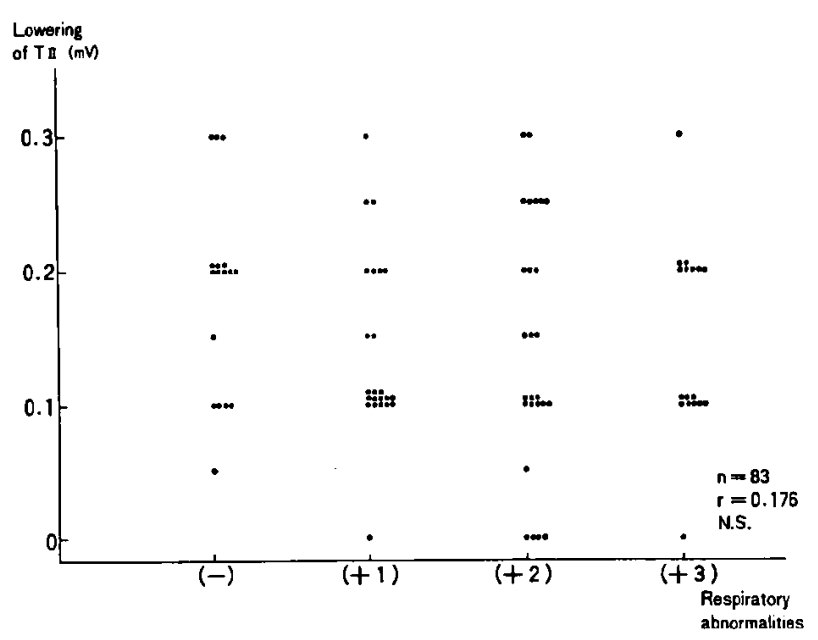

Fig. 6. Relationship between the lowering of $T_{I I}$ in the standing position and abnormalities in the respiratory curve.

correlation between the lowering of $\mathrm{T}_{\mathrm{II}}$ in the standing position and the respiratory curve, however, was not significant statistically (Fig. 6).

The relationships between these 3 parameters and the psychological tests are summarized in Table II. The normal psychological test group had smaller hearts and few abnormal findings in the respiratory curve, while the abnormal psychological test group had normal-sized hearts and many abnormalities in the respiratory curve.

As for the mutual relationship between cardiovascular parameters, no significant difference was found between the BP, CI, and SVR of the normal and the abnormal CMI groups when measured in the supine position (Table 
Table II. Relationship of CTR, the Lowering of $\mathrm{T}_{\mathrm{I}}$ in the Standing Position, the Respiratory Gurve, and Psychological Tests

\begin{tabular}{|c|c|c|c|c|}
\hline & \multicolumn{2}{|c|}{ CMI } & \multicolumn{2}{|c|}{ Y-G } \\
\hline & Normal & Abnormal & Normal & Abnormal \\
\hline CTR & small & normal & small & normal \\
\hline Lowering of $T_{I I}$ & remarkable & not remarkable & \multicolumn{2}{|c|}{ no difference } \\
\hline Respiratory abnormalities & few & many & few & many \\
\hline
\end{tabular}

Table III. Relationship between Psychological Tests and Cardiovascular Parameters Measured in the Supine Position

\begin{tabular}{|c|c|c|c|c|c|c|c|}
\hline & \multirow{2}{*}{$\begin{array}{l}\text { Total } \\
\text { cases } \\
(n=83)\end{array}$} & \multicolumn{3}{|c|}{ CMI } & \multicolumn{3}{|c|}{$Y-G$} \\
\hline & & $\begin{array}{l}\text { normal } \\
(\mathrm{n}=35)\end{array}$ & & $\begin{array}{l}\text { abnormal } \\
(n=48)\end{array}$ & $\begin{array}{l}\text { narmal } \\
(\mathrm{n}=71)\end{array}$ & & $\begin{array}{c}\text { abnormal } \\
(n=12)\end{array}$ \\
\hline $\begin{array}{l}\text { Systolic blood pressure } \\
(\mathrm{mmHg})\end{array}$ & $113 \pm 13$ & $112 \pm 14$ & ns & $114 \pm 12$ & $112 \pm 13$ & ns & $118 \pm 12$ \\
\hline $\begin{array}{l}\text { Mean blood pressure } \\
(\mathrm{mmHg})\end{array}$ & $85 \pm 10$ & $83 \pm 10$ & ns & $86 \pm 9$ & $83 \pm 10$ & $\mathrm{p}<0.05$ & $91 \pm 5$ \\
\hline $\begin{array}{l}\text { Cardiac index } \\
\left(\mathrm{L} / \mathrm{min} / \mathrm{M}^{2}\right)\end{array}$ & $4.0 \pm 1.0$ & $3.9 \pm 0.9$ & ns & $4.2 \pm 1.0$ & $4.0 \pm 0.9$ & $\mathrm{p}<0.05$ & $4.6 \pm 1.0$ \\
\hline $\begin{array}{l}\text { Systemic vascular } \\
\text { resistance } \\
\left(\text { dynes } \cdot \mathrm{sec} \cdot \mathrm{cm}^{-5}\right)\end{array}$ & $1750 \pm 450$ & $1802 \pm 504$ & $\mathrm{~ns}$ & $1729 \pm 403$ & $1780 \pm 466$ & ns & $1644 \pm 360$ \\
\hline
\end{tabular}

III). However, the BPm and CI of the abnormal Y-G group were significantly higher than those of the normal Y-G group, although the measured values remained within the normal range.

(6) Relationship between psychological tests and the effects of posture on cardiovascular parameters

In order to differentiate orthostatic hypotension from NCA, the relationship between psychological test results and the responses of the cardiovascular parameters to changes in posture was examined. As illustrated in Table IV, the ratios of change in BPm measured in the supine position and both immediately and $10 \mathrm{~min}$ after postural change to a standing position were significantly larger in the normal Y-G group than in the abnormal one. In addition, values of CI measured immediately after standing and 10 min later were significantly lower in the normal Y-G group than in the abnormal group, and values of SVR measured $10 \mathrm{~min}$ after standing were significantly higher in the normal Y-G group than in the abnormal group. Although we also examined the relationship between the postural changes in the cardiovascular parameters, the lowering of $T_{I I}$ in the standing position, CTR and respiratory 


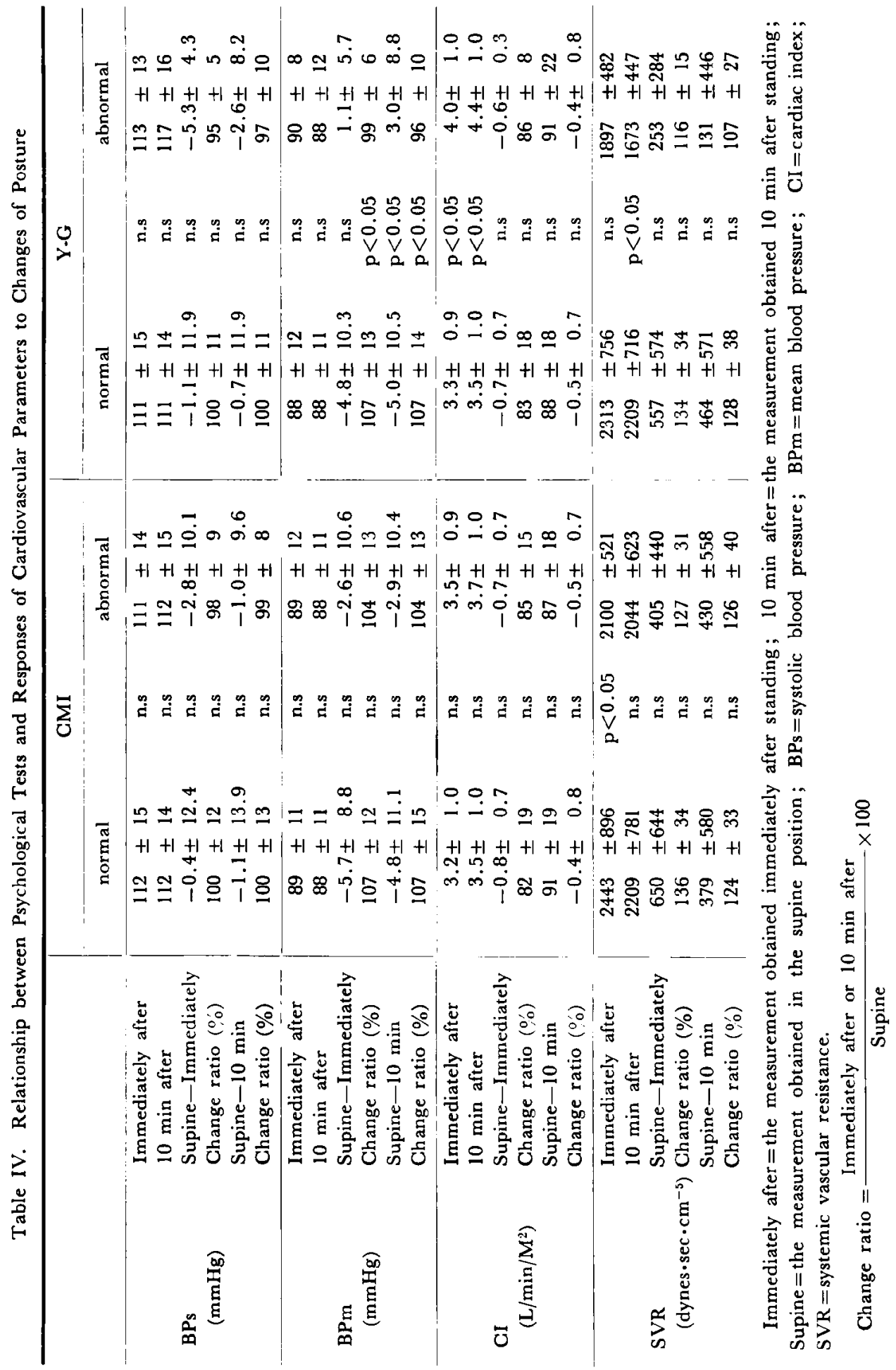


abnormalities, no significant difference was observed in either the supine or the standing positions.

\section{Discussion}

Purpose of the use of psychological tests for the study of $\mathcal{N} C A$

As previously mentioned, it is not easy to differentiate NCA from cardiac neurosis. Cohen et al $^{91}$ stated that NCA was included in the psychoneurosis as a result of psychological tests. Since NCA and psychoneurosis, particularly the anxiety type, are very difficult to distinguish, White included both NCA and cardiac neurosis in the title of his paper. ${ }^{5}$ However, it is certain that some cases of NCA have cardiovascular complaints which are not related to psychological factors. In such cases, subjective and objective symptoms can only be ascribed to somatic factors.

In order to investigate this problem, it is appropriate to use patients who have cardiovascular complaints, in the absence of organic cardiac disorders to determine whether cases without psychological abnormalities have cardiovascular abnormalities.

Comparison of the cardiovascular parameters of the normal and abnormal psychological test groups

Patients with normal CMI results displayed a smaller heart size, more marked lowering of $T_{I I}$ in the standing position and a greater increase in SVR immediately after standing than those with abnormal CMI scores. Studies on the response of cardiovascular parameters to change of posture revealed that the abnormal Y-G group displayed a smaller difference between BPm measured in the supine position and both immediately or $10 \mathrm{~min}$ after standing, and a larger difference in SVR measured before and after standing than the normal group. These differences indicate that the changes observed in the psychologically abnormal group are closer to the normal physiological state than those in the psychologically normal group.

Changes in cardiovascular parameters in the normal psychological test group

Thirty-five of the 83 cases studied had normal results on the psychological tests. With the exception of only 3 cases, the cases with normal psychological test results displayed some cardiovascular abnormalities in blood pressure, pulse rate, CI, $\mathrm{T}_{\mathrm{II}}$, and CTR. In other words, almost all the patients with normal psychological test results, who displayed cardiovascular complaints without organic abnormalities, had abnormalities in one or more of the cardiocascular parameters which, with the exception of CTR, are regarded as functional factors. These data suggest that the cardiovascular complaints of the patients without psychological abnormalities can be attributed to somatic 
factors, namely abnormalities in the cardiovascular parameters.

\section{Relationship between psychological tests and respiratory abnormalities}

Abnormalities in respiration were seen more frequently in patients with abnormal psychological test results than in those with normal results. This indicates that abnormal respiration is related to abnormal GMI or Y-G test results. Kimura ${ }^{2}$ has stated that an abnormal respiratory curve is regarded as one of the objective characteristics of NCA. However, if only those patients with normal psychological test results are regarded as suffering from NCA, abnormal respiratory curves seem to be a symptom of cardiac neurosis (a kind of psychoneurosis) rather than as a characteristic of NCA.

According to Kimura, ${ }^{10)}$ the lowering of $T_{I I}$ in the standing position and the abnormal respiratory curve seen in NCA were improved by the administration of ergotamine tartrate. In our present study, though, no specific relationship was found between the lowering of $\mathrm{T}_{\mathrm{II}}$ in the standing position and the abnormal respiratory curve. The lowering of $\mathrm{T}_{\mathrm{II}}$ in the standing position was more prominent in patients with in CMI-group I, who were regarded as normal psychologically. Furthermore, from the viewpoint of the relationship between the results of psychological tests and the respiratory curve, an abnormal respiratory curve and the lowering of $T_{I I}$ in the standing position appeared to be of a different nature.

Relationship between heart size and cardiovascular parameters

The group with normal psychological test results showed one or more cardiovascular abnormalities. In particular, a small CTR was observed in many instances in this group. The average CTR was $42 \%$, which was smaller than the lower limit of the normal range reported by Comeau et al. ${ }^{11)}$ Master $^{4}$ ) emphasized that the incidence of a small heart is much higher in NCA than in both other types of functional heart disease and the general population, and stated that NCA is not a functional disorder but an organic disease due to a small heart. From this point of view, he proposed the term "small heart syndrome" for this condition.

On the other hand, Friedman ${ }^{12)}$ and Carlotti et al ${ }^{13)}$ stated that normal controls had an average CTR of $40 \%$, and no difference was observed between the CTRs of NCA patients and the normal controls. Generally speaking, though, the value of $40 \%$ they obtained for their normal controls was excessively small, even if all their controls were young people.

Master') ascribed the complaints of NCA patients with small hearts to the inadequate regulation of blood pressure and pulse rate at the time of exercise, diminished venous return and cardiac output when sitting, standing, and exercising, and to the resulting decrease in oxygen saturation of the arterial blood. However, Master did not actually measure these parameters. 
In our results, the only parameters which were related to CTR were a lowering of $\mathrm{T}_{\mathrm{II}}$ in the standing position and SVR, while CO (CI), BP, and pulse rate, which are considered to be the causes of the cardiovascular complaints in NCA, had no correlation with CTR. However, these data suggest that there is some kind of relationship between small heart size and cardiovascular complaints. Since cardiovascular parameters other than CTR are always fluctuating, complaints in patients with a small heart may be the result of insufficient regulation of the circulatory system during standing or exercising, thus producing the symptoms of NCA.

In summary, a majority of the patients without psychological abnormalities but with the complaints of NCA have small hearts. However, we conclude that not all complaints or symptoms can be ascribed to small hearts, but that the symptoms produced by a small heart may present similar symptoms to NCA, although Master proposed that all cases of NCA be described as small heart syndrome. In other words, a small heart may be one of the signs of NCA.

\section{REFERENGES}

1. Christie RV: Some types of respiration in the neurosis. Quart J Med 4: 427, 1935

2. Kimura E: Respiratory findings in the neurocirculatory asthenia. Jpn Heart J 1: 72, 1960

3. Kimura E, Takahashi K, Ichikawa A, Kusachi N: Abnormal respiration and unusual movement of the diaphragm in patients with neurocirculatory asthenia. Am J Med Sci 265: 205, 1973

4. Master AM: Neurocirculatory asthenia due to small heart. Med Clin North Am 28: 577, 1944

5. White PD: Heart Disease, 4th Ed, MacMillan Co, New York, p 578, 1959

6. Brodman K, Erdmann AJ, Lorge I, Wolf HG: The Cornell medical index. An adjunct to medical interview. JAMA 140: 530, 1949

7. Friedberg CK: Diseases of the Heart, 3rd Ed, WB Saunders Co, Philadelphia \& London, p 1722, 1966

8. Fukamachi T: Study on Cornell Medical Index (Part II). Fukuoka Igaku Zasshi 50: 3001, 1959 (in Japanese)

9. Cohen ME, White PD, Johnson RE: Neurocirculatory asthenia, anxiety neurosis or the effort syndrome. Arch Intern Med 81 : 260, 1948

10. Kimura E, Obara F: Effect of ergotamine tartrate on cardiac neurosis (neurocirculatory asthenia). J Therapy 39: 462, 1957 (in Japanese)

11. Comeau WJ, White PD: A critical analysis of standard methods of estimating heart size from roentgen measurements. Am J Roentgenol Rad Ther 47: 665, 1942

12. Friedman $\mathbf{M}$ : Studies concerning the etiology and pathogenesis of neurocirculatory asthenia. III. The cardiovascular manifestation of neurocirculatory asthenia. Am Heart J 30: 478, 1945

13. Carlotti J, Cohen ME, White PD: The heart size in neurocirculatory asthenia, effort syndrome or anxiety neurosis. Am Heart J 36: 552, 1947 